PREPARED FOR THE U.S. DEPARTMENT OF ENERGY, UNDER CONTRACT DE-AC02-76CH03073

PPPL-3788

PPPL-3788

UC-70

Non-existence of Tokamak Equilibria with Negative Central Current

by

G.W. Hammett, S.C. Jardin, and B.C. Stratton

February 2003

M

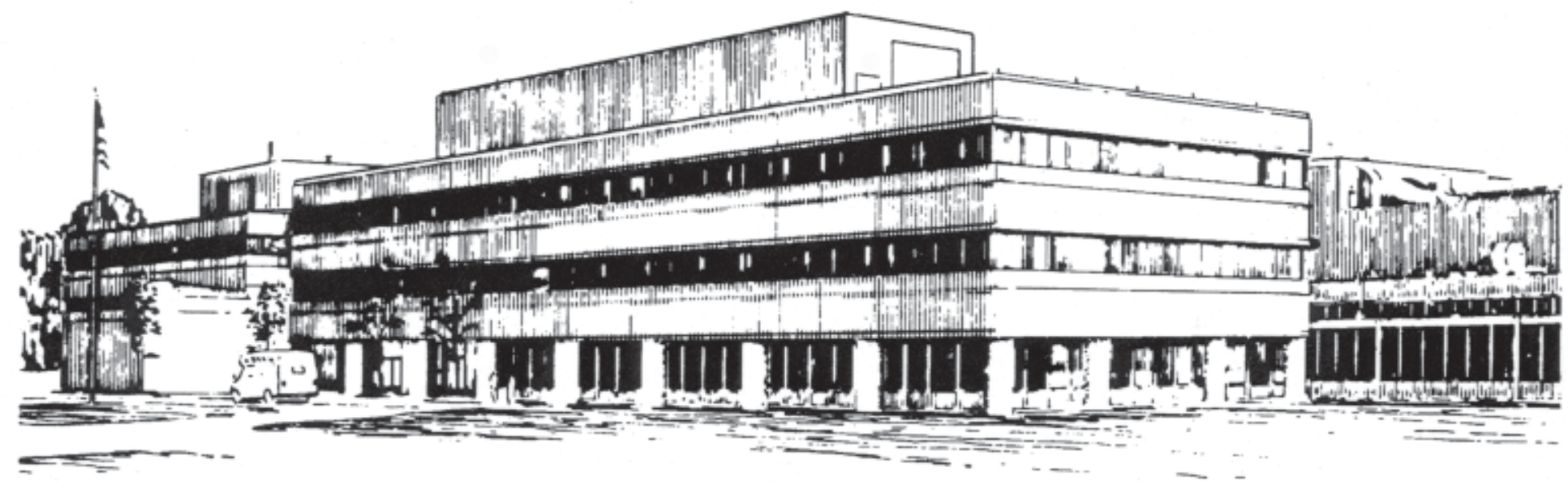

PRINCETON PLASMA PHYSICS LABORATORY PRINCETON UNIVERSITY, PRINCETON, NEW JERSEY 


\section{PPPL Reports Disclaimer}

This report was prepared as an account of work sponsored by an agency of the United States Government. Neither the United States Government nor any agency thereof, nor any of their employees, makes any warranty, express or implied, or assumes any legal liability or responsibility for the accuracy, completeness, or usefulness of any information, apparatus, product, or process disclosed, or represents that its use would not infringe privately owned rights. Reference herein to any specific commercial product, process, or service by trade name, trademark, manufacturer, or otherwise, does not necessarily constitute or imply its endorsement, recommendation, or favoring by the United States Government or any agency thereof. The views and opinions of authors expressed herein do not necessarily state or reflect those of the United States Government or any agency thereof.

\section{Availability}

This report is posted on the U.S. Department of Energy's Princeton Plasma Physics Laboratory Publications and Reports web site in Fiscal Year 2003. The home page for PPPL Reports and Publications is: http://www.pppl.gov/pub_report/

DOE and DOE Contractors can obtain copies of this report from:

U.S. Department of Energy

Office of Scientific and Technical Information

DOE Technical Information Services (DTIS)

P.O. Box 62

Oak Ridge, TN 37831

Telephone: (865) 576-8401

Fax: (865) 576-5728

Email: reports@adonis.osti.gov

This report is available to the general public from:

National Technical Information Service

U.S. Department of Commerce

5285 Port Royal Road

Springfield, VA 22161

Telephone: $1-800-553-6847$ or

(703) $605-6000$

Fax: (703) 321-8547

Internet: http://www.ntis.gov/ordering.htm 
PPPL-3788/Arxiv.org??

\title{
Non-existence of normal tokamak equilibria with negative central current
}

\author{
G. W. Hammett*, S. C. Jardin, and B. C. Stratton \\ Princeton Plasma Physics Laboratory \\ (Dated: Feb. 14, 2003, Submitted to Phys. Plasmas, rev. February 24, 2003)
}

\begin{abstract}
Recent tokamak experiments employing off-axis, non-inductive current drive have found that a large central current hole can be produced. The current density is measured to be approximately zero in this region, though in principle there was sufficient current drive power for the central current density to have gone significantly negative. Recent papers have used a large aspect-ratio expansion to show that normal MHD equilibria (with axisymmetric nested flux surfaces, non-singular fields, and monotonic peaked pressure profiles) can not exist with negative central current. We extend that proof here to arbitrary aspect ratio, using a variant of the virial theorem to derive a relatively simple integral constraint on the equilibrium. However, this constraint does not, by itself, exclude equilibria with non-nested flux surfaces, or equilibria with singular fields and/or hollow pressure profiles that may be spontaneously generated.
\end{abstract}

PACS numbers: $52.55 .-\mathrm{s}, 52.55 . \mathrm{Fa}$

\section{INTRODUCTION}

Tokamaks with reversed central magnetic shear (and thus low core current density) are of interest for at least two reasons: 1) internal transport barriers associated with reduced turbulence are often observed in them, leading to improved energy and particle confinement; and 2) they are the natural result of high beta operation and high bootstrap current fraction used to reduce noninductive current drive requirements for steady state operation. Both of these features could make reversed magnetic shear operation attractive for a tokamak reactor.

Recent experiments on $\mathrm{JET}^{1-4}$ and JT-60U ${ }^{5,6}$ have pushed the core current density to very low values using off-axis, non-inductive current drive. Large central current holes (regions of nearly zero current density) are produced because off-axis, non-inductive current drive in the same direction as the Ohmic current induces a back electromotive force inside the non-inductive current drive radius that decreases the core current density.

An interesting feature of current hole discharges is that the core current density is approximately zero (within Motional Stark Effect diagnostic measurement errors), even though there is often sufficient current drive power that the core current could in principal go significantly negative $^{2,3}$ (negative relative to the direction of the total plasma current). Recent non-linear toroidal resistive MHD simulations ${ }^{2,7}$ predict that current hole discharges undergo rapid $n=0$ reconnection events (axisymmetric sawteeth) that clamp the core current near zero. More generally, this reconnection occurs whenever the current density profile is such that the rotational transform, $\iota$, goes to zero on any surface in the plasma (this includes the case where the current density on-axis is positive, but the current profile goes sufficiently negative somewhere off-axis that the total current enclosed by some flux sur-

\footnotetext{
*Electronic mail: hammett@princeton.edu
}

face vanishes). Reduced MHD simulations in cylindrical geometry have also shown that $n=0$ resistive kink instabilities can clamp the core current density at zero when it attempts to go negative. ${ }^{8}$ Breslau et al. ${ }^{7}$ and Stratton et al. $^{2}$ stated that a second-order, large aspect ratio expansion of the MHD equations indicates that a normal toroidal equilibrium is not possible if $\iota$ crosses through 0 at some radius. (They also stated that a more general proof is needed, which we provide here.) A recent paper by Chu and Parks ${ }^{9}$ used a second order aspect ratio expansion to prove that a normal equilibrium with a peaked pressure profile is not possible with negative core current. They extended the analysis to provide matching conditions at the boundary of a central region with no current and no pressure gradient, showing explicitly that current hole equilibria are theoretically possible (with zero, but not negative, current).

This paper extends some of these results to arbitrary aspect ratio, employing a relatively simple constraint based on a version of the virial theorem to show that a "normal" toroidal MHD equilibrium (with axisymmetric nested flux surfaces around a single magnetic axis, non-singular continuous fields, and a monotonic peaked pressure profile) is not possible with negative core current. Or more generally, a normal equilibrium is not possible if the toroidal current enclosed by any flux surface goes negative relative to the direction of the total plasma current, so that there is an $\iota=0$ surface somewhere in the plasma where the poloidal field vanishes (the null surface). Though the starting point of this analysis is based on well-known equations, they are often specialized to large aspect ratio or simplified geometry, while the present analysis is more general.

However, the virial constraint does not necessarily eliminate the possibility of more exotic equilibria, such as with non-nested flux surfaces with islands, or with singular fields and/or off-axis peaks in the pressure profiles that may be spontaneously generated by the plasma near the null surface. Some examples are considered here. In this paper we investigate the consequences of only one in- 
tegral constraint on equilibria, while there can be other constraints that further limit the types of theoretically possible or experimentally realizable equilibria ${ }^{9}$.

The non-existence of normal equilibria with negative core current, and/or the rapid axisymmetric sawteeth that are predicted to occur if the enclosed current goes negative, may also explain the results of other experiments, such as the low efficiency seen in some electron cyclotron counter current drive experiments ${ }^{6,10}$.

\section{DERIVATION}

The MHD equilibrium equation $\nabla p=\boldsymbol{j} \times \boldsymbol{B} / c$ can be written as

$$
0=-\nabla\left(p+\frac{B^{2}}{8 \pi}\right)+\frac{1}{4 \pi}(\boldsymbol{B} \cdot \nabla) \boldsymbol{B}
$$

One common use of the virial theorem is to take the inner product of this equation with the position vector $\boldsymbol{x}$ and integrate over all space to show that an isolated MHD equilibrium can not exist by itself (unless there are physical coils or gravity to provide overall force balance). ${ }^{11,12}$ Here we use a version of the virial theorem that can be used to derive the Shafranov shift ${ }^{13}$, by focusing on radial force balance of axisymmetric equilibria in cylindrical coordinates $(R, Z, \phi)$. Taking the inner product of Eq. (1) with $\boldsymbol{R}=R \hat{\boldsymbol{R}}$, the radial vector in cylindrical geometry, and integrating over space out to some flux surface of volume $V$, gives

$$
0=-\int d V \boldsymbol{R} \cdot \nabla\left(p+\frac{B^{2}}{8 \pi}\right)+\frac{1}{4 \pi} \int d V \boldsymbol{R} \cdot(\boldsymbol{B} \cdot \nabla) \boldsymbol{B}
$$

For the second integral we use the identity $\boldsymbol{R} \cdot(\boldsymbol{B} \cdot \nabla) \boldsymbol{B}=$ $\nabla \cdot(\boldsymbol{B} \boldsymbol{R} \cdot \boldsymbol{B})-B_{R}^{2}-B_{\phi}^{2}$. The integral of $\nabla \cdot(\boldsymbol{B} \boldsymbol{R} \cdot \boldsymbol{B})$ vanishes because $\boldsymbol{B} \cdot d \boldsymbol{S}=0$ on a flux surface. The first integral can be integrated by parts using $\nabla \cdot \boldsymbol{R}=2$, so that Eq. (2) becomes

$$
\begin{aligned}
0= & -p(\rho) \int d \boldsymbol{S} \cdot \boldsymbol{R}-\int d \boldsymbol{S} \cdot \boldsymbol{R} \frac{B^{2}}{8 \pi} \\
& +2 \int d V\left(p+\frac{B^{2}}{8 \pi}\right)-\frac{1}{4 \pi} \int d V\left(B_{R}^{2}+B_{\phi}^{2}\right) \\
= & -p \int d \boldsymbol{S} \cdot \boldsymbol{R}-\int d \boldsymbol{S} \cdot \boldsymbol{R} \frac{B^{2}}{8 \pi}+2 \int d V\left(p+\frac{B_{Z}^{2}}{8 \pi}\right),
\end{aligned}
$$

where $p=p(\rho)$ is the pressure at the surface labeled by $\rho$ enclosing the volume $V(\rho)$, and $B_{Z}$ is the vertical magnetic field. For the first surface integral we can use Gauss' theorem to write $\int d \boldsymbol{S} \cdot \boldsymbol{R}=\int d V \nabla \cdot \boldsymbol{R}=2 V$. For the second surface integral, we use $d \boldsymbol{S}=2 \pi R \hat{\boldsymbol{\phi}} \times$ $d \vec{\ell}$, where $d \vec{\ell}$ is a poloidal path length element along the surface, to write $d \boldsymbol{S} \cdot \boldsymbol{R}=2 \pi R^{2} \hat{\boldsymbol{Z}} \cdot d \vec{\ell}$ so $\int d \boldsymbol{S} \cdot \boldsymbol{R}=$ $2 \pi \oint R^{2} \hat{\boldsymbol{Z}} \cdot d \vec{\ell}=2 \pi \oint R^{2} d Z$. Since the toroidal field $B_{\phi} \propto$

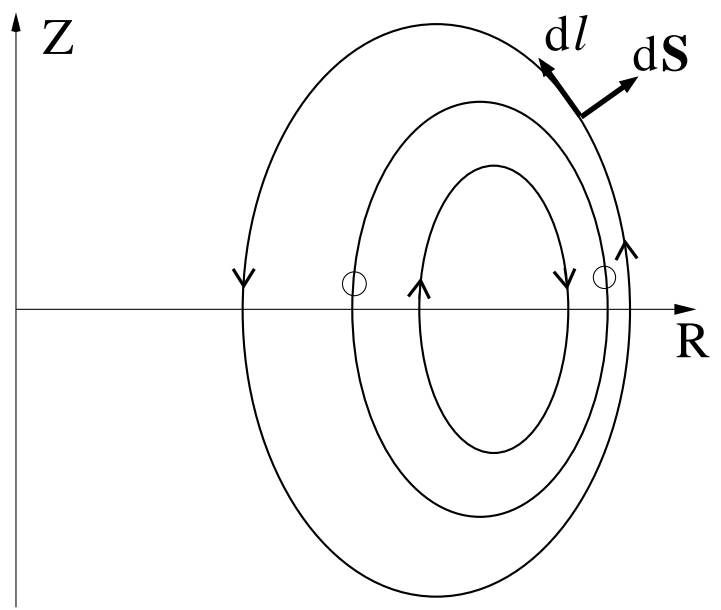

FIG. 1: Sketch of hypothetical equilibrium with nested flux surfaces and negative central current (relative to the total current), so that the poloidal field points clockwise near the axis, counterclockwise near the edge, and is zero on a flux surface in between. A normal equilibrium is not possible in this case (with a normal peaked pressure profile)

$1 / R$ in a flux surface, the $B_{\phi}^{2}$ contribution to this surface integral vanishes, and we have

$$
0=-p(\rho)-\frac{1}{V} \oint d \vec{\ell} \cdot \hat{\boldsymbol{Z}} \pi R^{2} \frac{B_{p o l}^{2}}{8 \pi}+\langle p\rangle+\left\langle\frac{B_{Z}^{2}}{8 \pi}\right\rangle
$$

where $\langle\ldots\rangle=\int d V \ldots / \int d V$ denotes a volume average, and $B_{\text {pol }}^{2}=B_{R}^{2}+B_{Z}^{2}$ is the poloidal field strength squared. The poloidal field can be written as $\boldsymbol{B}_{\text {pol }}=$ $\nabla \phi \times \nabla \psi=(\hat{\boldsymbol{\phi}} \times \nabla \rho)(\partial \psi / \partial \rho) / R$, where $\rho$ is a flux surface label. [While $\psi$ is also constant on a flux surface, there can be two surfaces with the same value of $\psi$ in the presence of negative central current, so it convenient to choose another flux surface label $\rho$, such as based on the enclosed volume or toroidal flux, to maintain monotonic labeling.] If the toroidal current near the magnetic axis is in the opposite direction as the total plasma current, then the poloidal field must reverse direction somewhere and there must be a null surface on which the poloidal magnetic field is everywhere zero, as shown in Fig. 1. Another way to see this is to note that the poloidal field is related to the enclosed toroidal current by $4 \pi I_{\phi}(\rho) / c=\oint d \vec{\ell} \cdot \boldsymbol{B}_{\text {pol }}=(\partial \psi / \partial \rho) \oint d \vec{\ell} \cdot \hat{\boldsymbol{\phi}} \times \nabla \rho / R$, so $B_{\text {pol }} \propto \partial \psi / \partial \rho=0$ on any flux surface that encloses zero toroidal current. This is also the flux surface on which the rotational transform $\iota=0$ (corresponding to the safety factor $q=\infty)$. [These arguments assume that $B_{p o l}$ is continuous and finite, we consider a singular exception in the next section.]

On such a flux surface where the poloidal field vanishes, the second term of Eq. (4) vanishes and we are left with constraint

$$
0=-p+\langle p\rangle+\left\langle\frac{B_{Z}^{2}}{8 \pi}\right\rangle
$$




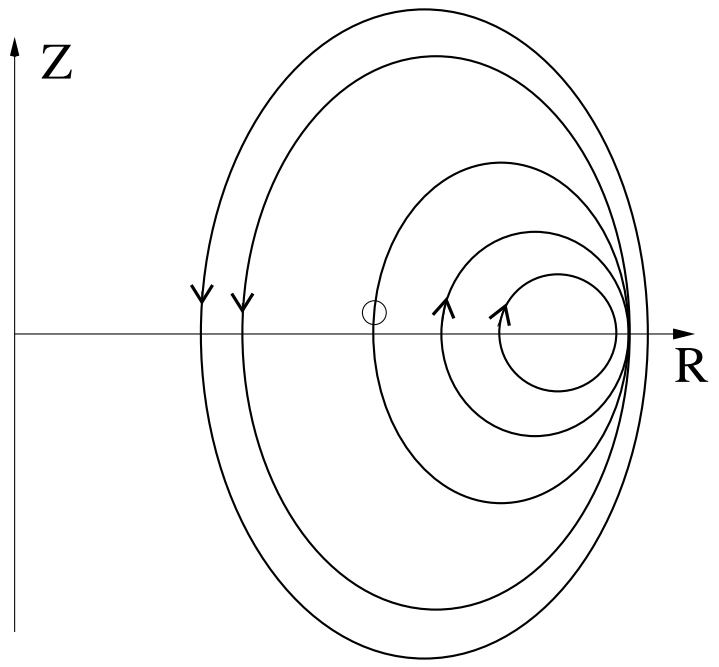

FIG. 2: Ideal MHD allows singular solutions where the poloidal field vanishes almost everywhere on a flux surface, so that the enclosed current $\propto \oint d \vec{\ell} \cdot \boldsymbol{B}_{\text {pol }}=0$ but $\oint d \ell B_{\text {pol }}^{2}$ is finite. Such an equilibrium could then in principle satisfy the integral force balance Eq. (4). In this case, adjacent flux surfaces approach one another at one point where the poloidal field becomes infinite, but this is an integrable singularity with finite energy. [All of these sketches are intended only to illustrate topology and are not precise.]

Since the last two terms are positive definite, the only way this equation can possibly be satisfied is if the pressure at this flux surface, $p$, is larger than the volumeaveraged pressure inside that flux surface, $\langle p\rangle$. I.e., the pressure profile must be hollow, and can't monotonically decrease with increasing $\rho$ as usual pressure profiles do.

This is in agreement with the result of Chu and Parks ${ }^{9}$, who also found that a normal equilibrium with a negative central current is not possible if the pressure profile is monotonically decreasing. These earlier results used a second-order large aspect ratio ordering while our derivation is valid for arbitrary aspect ratio. In other ways, their calculation goes beyond ours, as they have investigated additional constraints that can further limit the class of accessible equilibria.

\section{POSSIBLE ALTERNATE SOLUTIONS}

Here we consider several possible alternate solutions for satisfying force balance in equilibria. Each differs from "normal" equilibria in a different way.

The argument in the previous section applies rigorously only for nested flux-surfaces where the fields are continuous and finite. Within the framework of ideal MHD, in principle there could be a singular poloidal field on a flux surface such that $\oint d \vec{\ell} \cdot \boldsymbol{B}_{\text {pol }}=0$ so that this surface includes zero toroidal current, but $\int d \vec{\ell} \cdot \hat{\boldsymbol{Z}} \pi R^{2} B_{\text {pol }}^{2} /(8 \pi)$ is still finite and can contribute to Eq. (4) so that integrated force balance can be satisfied.

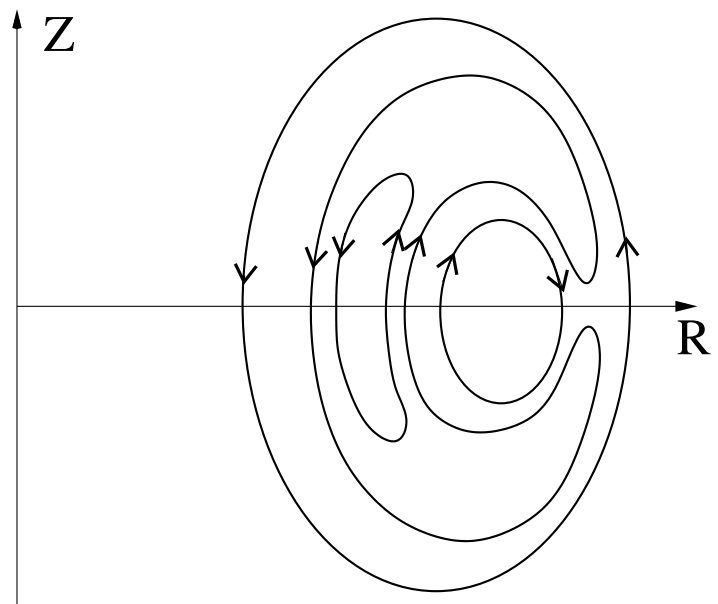

FIG. 3: An equilibrium with this topology of non-nested flux surfaces is not ruled out by the integral force balance Eq. (4). Note that the toroidal current in the inner part of the plasma is in the reverse direction from the total plasma current.

An example of such a field might be the limiting case $B_{\text {pol }} \propto|\nabla \psi| \sim C \exp \left(-\ell^{2} / w^{2}\right) / \sqrt{w}$. Then in the limit as $w \rightarrow 0$ we have zero toroidal current enclosed while still giving a finite contribution to the second term of Eq. (4). Although $B_{p o l}$ is becoming infinite at some point on the flux surface, it is an integrable singularity containing a finite amount of energy, and so could formally be considered as an admissible solution of ideal MHD. Since the spacing between two nearby flux surfaces labeled by poloidal flux $\psi_{1}$ and $\psi_{2}$ is given by $\Delta \approx\left(\psi_{2}-\psi_{1}\right) /|\nabla \psi|$ (except where second derivatives have to be considered), there will be flux surfaces with finite separation at some places which will approach one another at the singular point where $|\nabla \psi| \rightarrow \infty$. The topology of this configuration is illustrated in Fig. 2. [This sketch is intended only to illustrate the topology of a possible solution which satisfies the integral force balance constraint, Eq. (4). An actual detailed solution that would satisfy force balance locally at all points is left for future work.] Note that not only is the poloidal field infinite at the singular point, it must also flip signs from $+\infty$ to $-\infty$ in the limit as the singular point is approached radially from opposite directions. Of course this configuration is strongly susceptible to magnetic reconnection when resistivity is included, consistent with the interpretation of rapid reconnection observed in simulations ${ }^{2,7}$. If finite resistivity is included, then the singularity in the field is smoothed out and loss of equilibrium can help drive reconnecting flows.

Another possible way of satisfying integrated force balance with negative central current, while keeping the field finite and continuous, is if the flux surfaces are nonnested. An example is shown in Fig. 3, which is similar to the intermediate configurations observed during some toroidal simulations of axisymmetric reconnection in negative central current plasmas (for example, Fig. 11 of Ref. 2, though in other cases they see islands with 
higher poloidal mode numbers). This case has two axisymmetric islands and the poloidal field is finite almost everywhere (except at the two magnetic axes and at the $\mathrm{X}$-point) and so in principle can be arranged to give a negative contribution to the second term of Eq. (4) to satisfy integrated force balance. This is related to the role in a normal equilibrium of the Shafranov shift, which provides a larger value of $R^{2} B_{p o l}^{2}$ on the outer part of a flux surface than on the inner part, so that the second term in Eq. (4) is negative. The X-point of a non-nested equilibrium might not be on the low-field side, and another possible equilibrium might be obtained by rotating Fig. 3 by $180^{\circ}$ and shifting the spacing between flux surfaces so that the integral of $R^{2} B_{p o l}^{2}$ on the outer part of the flux surface is again larger than on the inner part. One or the other of these configurations may be an unstable equilibrium and prefer to flip to the other orientation. [Takizuka ${ }^{14}$ earlier proposed another possible nonnested equilibria with negative central current, involving $(m=2, n=0)$ islands, while the example we discussed here has an $(m=1, n=0)$ island.]

Another way of thinking about ideal MHD equilibria is to modify the time-dependent ideal MHD equations to include viscosity and parallel thermal conduction while retaining the ideal Ohm's law ${ }^{12}$. Since viscosity should eventually damp the velocity $\boldsymbol{u}$ to zero, and parallel thermal conduction will lead to $\boldsymbol{B} \cdot \nabla p=0$, the dissipative terms vanish in a stationary steady state and the solution is also an ideal MHD equilibrium. One can then start with any arbitrary initial configuration of the magnetic field (which can be assumed to be nested flux surfaces) with arbitrary initial profiles, as functions of toroidal flux $\Phi$, for the rotational transform $\iota(\Phi)$ and the adiabatic parameter $\mu(\Phi)=p / n^{\Gamma}$ (where $p(\Phi)$ is the pressure profile, $n(\Phi)$ is the density profile, and $\Gamma$ is the ratio of specific heats). Since this initial condition is not necessarily an equilibrium, flows will be driven and the plasma will move about, perhaps oscillating for a while. But it seems reasonable to assume that the viscosity will eventually damp out the oscillations and the plasma will settle into an equilibrium configuration while conserving $\iota(\Phi)$ and $\mu(\Phi)$. (The motions are assumed to be constrained to be axisymmetric to find such an equilibria. This approach to equilibria of course does not address the issue of stability, and these equilibria might then be unstable to symmetry-breaking perturbations.)

This was the logic that motivated the flux-conserving tokamak equilibria concepts ${ }^{12}$ that showed that there is formally no equilibrium limit on the pressure in a tokamak, since as the pressure increases, the Shafranov shift and the current can also increase to provide sufficient poloidal magnetic field on the outboard side to provide force balance.

Presumably this procedure would also find an equilibrium even if the rotational transform changed sign so that there was a null flux surface where $\iota=0$. In some cases with certain initial conditions, it might be possible for the plasma to spontaneously adjust flux surfaces near the null $\iota$ surface to produce a local peak in the pressure profile that can satisfy Eq. (5). [We have focussed on the consequences of only one integral constraint that rules out "normal" equilibria with negative central current, and there can be other constraints that would further limit the practical accessibility of such hollow pressure equilibria $^{9}$.] The more typical case is probably that the equilibrium that is approached will have a singular structure, such as in Fig. 2, in order to satisfy Eq. (4). [This is similar to studies showing that the nonlinear saturation of an internal kink mode approaches a neighbouring equilibrium state with singular currents ${ }^{15,16}$.] These singular or near-singular states will be subject to strong reconnection if a small amount of resistivity is introduced, and the change in topology may dominate what happens. Realistic evaluations of what happens may depend on fully including various dissipation mechanisms (thermal, momentum, and particle anisotropic transport driven by small scale turbulence and collisional effects, as well as resistivity, current drive, heating and loss mechanisms). We leave detailed investigation of these issues to other work.

\section{RELATION TO THE SHAFRANOV SHIFT}

For completeness, we show the relation of Eq. (4) to usual expressions for the Shafranov shift. The second term of Eq. (4) can be written as

$T_{2}=-\frac{\pi}{V} \oint d \vec{\ell} \cdot \hat{Z} R^{2} \frac{B_{p o l}^{2}}{8 \pi}=-\frac{\pi}{V} \frac{(\partial \psi / \partial \rho)^{2}}{8 \pi} \oint d \vec{\ell} \cdot \hat{Z}|\nabla \rho|^{2}$

At this point, many previous calculations specialize to a large aspect ratio expansion and/or to specified shapes for the flux surfaces. For example, assume shifted circular flux surfaces with $R(\rho, \theta)=R_{0}-\Delta(\rho)+\rho \cos \theta$ and $Z(\rho, \theta)=\rho \sin \theta$, where $\rho$ has now been chosen to be the minor radius of the flux surface, and $\Delta$ is the Shafranov shift. It can be shown that $|\nabla \rho|^{2}=1 /\left(1-\Delta^{\prime} \cos \theta\right)^{2}$, where $\Delta^{\prime}=d \Delta / d \rho$. Defining $\partial \psi / \partial \rho=B_{p 0}(\rho)\left(R_{0}-\Delta\right)$, we have $B_{p o l}=B_{p 0}\left[R_{0}-\Delta\right] /\left[R\left(1-\Delta^{\prime} \cos \theta\right)\right]$ (this would be exact if the flux surfaces really were shifted circles), and $T_{2}$ becomes

$$
T_{2}=-\frac{B_{p 0}^{2}}{8 \pi} \frac{R_{0}-\Delta}{r} \frac{\Delta^{\prime}}{\left(1-\Delta^{\prime}\right)^{3 / 2}}
$$

where we have used $\int d \theta \cos \theta /\left(1-\Delta^{\prime} \cos \theta\right)^{2}=2 \pi \Delta^{\prime} /(1-$ $\left.\Delta^{\prime}\right)^{3 / 2}$. Inserting this into Eq. (4) yields

$$
\frac{\Delta^{\prime}}{\left(1-\Delta^{\prime}\right)^{3 / 2}}=\frac{r}{R_{0}-\Delta} \frac{8 \pi}{B_{p 0}^{2}}\left(\langle p\rangle-p+\left\langle\frac{B_{Z}^{2}}{8 \pi}\right\rangle\right)
$$

Evaluating this at the plasma edge, where $p=0$, in the large aspect ratio limit, gives the familiar form $\Delta^{\prime}=$ $\left(r / R_{0}\right)\left(\beta_{\text {pol }}+\ell_{i} / 2\right)$, where $\beta_{\text {pol }}$ is the poloidal beta and $\ell_{i}$ is the internal inductance per unit length. The nonlinear 
form of the left-hand side of Eq. (8) has the nice property of insuring that the flux surfaces are well behaved and don't cross $\left(\left|\Delta^{\prime}\right|<1\right)$ for arbitrarily high $\beta_{\text {pol }}$, though this equation only rigorously applies if the flux surfaces remained shifted circles, which breaks down at high beta.

\section{SUMMARY}

We have presented a relatively simple integral constraint on toroidally axisymmetric MHD equilibrium that shows that a normal equilibrium (with nested magnetic flux surfaces, non-singular fields, and a peaked pressure profile that falls monotonically with radius) can not exist if the toroidal current inside any flux surface is negative relative to the total plasma current. This generalizes previous results ${ }^{2,7,9}$ to arbitrary aspect ratio.

However, the integral constraint does not necessarily prevent negative central current equilibria with nonnested or singular magnetic flux surfaces. Possible examples of this are shown in Figs. 2 and 3. A plasma with nested flux surfaces and negative central current that is initially out of equilibrium could presumably move towards an equilibrium, though it seems most likely that this new equilibrium would have singular or near-singular fields and thus would be subject to strong reconnection ${ }^{2,7}$ if finite resistivity is introduced, changing the topology. There can also be other constraints that limit the accessible class of alternate equilibria ${ }^{9}$. One might be able to understand the structure of some of these possible solutions in the vicinity of the null surface as a boundary layer analysis of a shock-like solution. But a realistic evaluation of such scenarios would require including finite crossfield transport, viscosity, resistivity, and FLR effects. We leave these issues to future work. Other interesting questions to consider are whether such "non-normal" MHD equilibria are stable to ideal and/or resistive MHD modes and/or are experimentally accessible.

\section{Acknowledgments}

We thank Drs. Joshua Breslau, Ming-Sheng Chu, Nathaniel Fisch, Nikolai Gorelenkov, Don Monticello, Paul Parks, Emilia Solano, and Leonid Zakharov for helpful discussions on these topics. GWH also thanks Dr. Paul Rutherford for teaching a course that covered a virial theorem approach to the Shafranov shift. Supported by DOE Contract \# DE-AC02-76CH03073.
1 N. C. Hawkes, B. C. Stratton, T. Tala, C. D. Challis, G. Conway, R. DeAngelis, C. Giroud, J. Hobirk, E. Joffrin, P. Lomas, et al., Phys. Rev. Lett. 87, 115001 (2001).

2 B. C. Stratton, J. A. Breslau, R. V. Budny, S. C. Jardin, W. Park, H. R. Strauss, L. E. Zakharov, B. Alper, V. Drozdov, N. C. Hawkes, et al., Plasma Phys. Controlled Fusion 44, 1127 (2002).

3 N. C. Hawkes, Y. Andrew, C. D. Challis, R. DeAngelis, V. Drozdov, J. Hobirk, E. Joffrin, P. Lotte, D. Mazon, E. Rachlew, et al., Plasma Phys. Control. Fusion 44, 1105 (2002).

4 B. C. Stratton, N. C. Hawkes, G. T. A. Huysmans, J. A. Breslau, L. E. Zakharov, B. Alper, R. V. Budny, C. D. Challis, R. Deangelis, V. Drozdov, et al., Proc. of the 19th IAEA Fusion Energy Conference (October 2002, Lyon, France), paper no. IAEA-CN-94/EX/C3-1Rb (2002).

5 T. Fujita, T. Oikawa, T. Suzuki, S. Ide, Y. Sakamoto, Y. Koide, T. Hatae, O. Naito, A. Isayama, N. Hayashi, et al., Phys. Rev. Lett. 87, 245001 (2001).

6 Y. Miura, T. Fujita, T. Oikawa, T. Suzuki, S. Ide, Y. Sakamoto, Y. Koide, T. Hatae, O. Naito, A. Isayama, et al., Proc. of the 19th IAEA Fusion Energy Conference (October 2002, Lyon, France), paper no. IAEA-CN94/EX/C3-1Ra (2002).

7 J. A. Breslau, S. C. Jardin, and W. Park (2003), "Simu- lation studies of the role of reconnection in the "current hole" experiments in the Joint European Torus", accepted for publication in Phys. Plasmas.

8 G. T. A. Huysmans, T. C. Hender, N. C. Hawkes, and X. Litaudon, Phys. Rev. Lett. 87, 245002 (2001).

9 M. S. Chu and P. B. Parks, Phys. Plasmas 9, 5036 (2002).

10 E. Westerhof, R. W. Polman, G. M. D. Hogeweij, J. Lok, E. Min, A. A. M. Oomens, and F. C. Schuller, Fus. Eng. and Design 53, 259 (2001).

11 V. D. Shafranov, in Reviews of Plasma Physics, edited by M. A. Leontovich (Consultants Bureau., 1966), vol. 2.

12 J. P. Freidberg, Ideal Magnetohydrodynamics (Plenum Press, 1987).

13 P. H. Rutherford (1981), private communication. The derivation of Eqs. 1-3 closely follows a virial theorem approach to the Shafranov shift, developed by Dr. Rutherford in his lecture notes for a course on tokamaks at Princeton University.

14 T. Takizuka, J. Plasma Fusion Res. 78, 1282 (2002).

15 W. Park, D. A. Monticello, R. B. White, and S. C. Jardin, Nucl. Fus. 20, 1181 (1980).

16 M. N. Rosenbluth, R. Y. Dagazian, and P. H. Rutherford, Phys. Fluids 16, 1894 (1973). 


\section{External Distribution}

Plasma Research Laboratory, Australian National University, Australia

Professor I.R. Jones, Flinders University, Australia

Professor João Canalle, Instituto de Fisica DEQ/IF - UERJ, Brazil

Mr. Gerson O. Ludwig, Instituto Nacional de Pesquisas, Brazil

Dr. P.H. Sakanaka, Instituto Fisica, Brazil

The Librarian, Culham Laboratory, England

Mrs. S.A. Hutchinson, JET Library, England

Professor M.N. Bussac, Ecole Polytechnique, France

Librarian, Max-Planck-Institut für Plasmaphysik, Germany

Jolan Moldvai, Reports Library, MTA KFKI-ATKI, Hungary

Dr. P. Kaw, Institute for Plasma Research, India

Ms. P.J. Pathak, Librarian, Insitute for Plasma Research, India

Ms. Clelia De Palo, Associazione EURATOM-ENEA, Italy

Dr. G. Grosso, Instituto di Fisica del Plasma, Italy

Librarian, Naka Fusion Research Establishment, JAERI, Japan

Library, Plasma Physics Laboratory, Kyoto University, Japan

Research Information Center, National Institute for Fusion Science, Japan

Dr. O. Mitarai, Kyushu Tokai University, Japan

Library, Academia Sinica, Institute of Plasma Physics, People's Republic of China

Shih-Tung Tsai, Institute of Physics, Chinese Academy of Sciences, People's Republic of China

Dr. S. Mirnov, TRINITI, Troitsk, Russian Federation, Russia

Dr. V.S. Strelkov, Kurchatov Institute, Russian Federation, Russia

Professor Peter Lukac, Katedra Fyziky Plazmy MFF UK, Mlynska dolina F-2, Komenskeho Univerzita, SK-842 15 Bratislava, Slovakia

Dr. G.S. Lee, Korea Basic Science Institute, South Korea

Institute for Plasma Research, University of Maryland, USA

Librarian, Fusion Energy Division, Oak Ridge National Laboratory, USA

Librarian, Institute of Fusion Studies, University of Texas, USA

Librarian, Magnetic Fusion Program, Lawrence Livermore National Laboratory, USA

Library, General Atomics, USA

Plasma Physics Group, Fusion Energy Research Program, University of California at San Diego, USA

Plasma Physics Library, Columbia University, USA

Alkesh Punjabi, Center for Fusion Research and Training, Hampton University, USA

Dr. W.M. Stacey, Fusion Research Center, Georgia Institute of Technology, USA

Dr. John Willis, U.S. Department of Energy, Office of Fusion Energy Sciences, USA

Mr. Paul H. Wright, Indianapolis, Indiana, USA 
The Princeton Plasma Physics Laboratory is operated by Princeton University under contract with the U.S. Department of Energy.

\author{
Information Services \\ Princeton Plasma Physics Laboratory \\ P.O. Box 451 \\ Princeton, NJ 08543
}

Phone: 609-243-2750

Fax: 609-243-2751

e-mail: pppl_info@pppl.gov

Internet Address: http://www.pppl.gov 